\title{
Étude de la dimension collective de l'usage des systèmes d'assistance à la conduite automobile en situation réelle : l'exemple du régulateur de vitesse conventionnel
}

Studying the collective dimension of driving assistance systems in real context: the conventional cruise control as example

\author{
Aupetit Samuel ${ }^{1,2, *}$, Riff Jacques ${ }^{1}$
}

\footnotetext{
${ }^{1}$ Laboratoire Activité Motrice et Adaptation Psycho Physiologique (AMAPP), Université d'Orléans, 2 allée du Château, BP 6237, 45062 Orléans Cedex 2, France

${ }^{2}$ Laboratoire Exploitation, Perception, Simulateurs et Simulations (LEPSIS), Institut National de Recherche sur les Transports et leur Sécurité (INRETS) / Laboratoire Central des Ponts et Chaussées (LCPC), 58 boulevard Lefebvre, 75731 Paris Cedex 15, France

* Doctorant financé par la Fondation MAIF et la Région Centre

Les idées contenues dans cet article n'engagent que leurs auteurs

Correspondance: Samuel Aupetit (E-mail : aupetit@inrets.fr, Tél. : +33(0)1 40436538 , Fax : +33(0)1404354 99)
} 


\section{Résumé}

Cet article présente une étude de la dimension collective de l'usage du régulateur de vitesse conventionnel, un système d'assistance à la conduite automobile très répandu dont la fonction est de conserver une allure prédéfinie par le conducteur. Dix conducteurs ont été suivis en «situation réelle» avec leur voiture personnelle munie de ce dispositif. Des données d'observation et de verbalisation (méthode biographique, entretien d'explicitation) ont été recueillies. Les résultats montrent (1) les contraintes que représentent les passagers non habitués à une vitesse régulée pour le conducteur, (2) la façon dont le régulateur de vitesse peut être envisagé comme élément de reproduction des jeux sociaux au sein du véhicule, (3) les difficultés de régulation de la vitesse dans le trafic entre véhicules équipés et non équipés, (4) les modifications de distance inter-véhicules et leurs conséquences sur la cohabitation entre automobilistes et (5) l'existence d'une dimension collective forte dans le processus d'appropriation du système.

Mots clés : Système d'aide à la conduite automobile ; Ergonomie ; Régulateur de vitesse conventionnel ; Activité réelle ; Dimension collective 


\begin{abstract}
This paper presents a study of the collective dimension of the use of conventional cruise control (CCC), a widespread driving assistance system, whose function is to maintain a preset speed by the driver. Ten French drivers were followed driving their own car equipped with CCC in real context. Observation and verbalisation (biographic method, explicitation interview) data were collected. The results show (1) the constraint represented by passengers unused to regulated speed for the driver, (2) the way that CCC may be considered as a reproduction tool of social play inside the vehicle, (3) the difficulties of speed control in the traffic between equipped and non equipped cars, (4) the changes in distance headway and their effect on drivers cohabitation and (5) the existence of a strong collective dimension in the appropriation process of the CCC.
\end{abstract}

Key words: Driving assistance system; Ergonomics; Conventional cruise control; Real activity; Collective dimension 
La recherche qui fait l'objet de cet article porte sur l'usage du régulateur de vitesse conventionnel, connu sous l'appellation américaine Conventional Cruise Control (CCC), un système d'assistance à la conduite automobile qui se charge de maintenir une allure de « croisière » enregistrée par le conducteur. Ce dispositif qui équipe aujourd'hui une grande partie du parc automobile français n'a pas donné lieu à un effort de recherche important sur son usage effectif. On ne dispose pas encore d'un corpus de données suffisant pour caractériser la manière dont les conducteurs utilisent réellement ce dispositif. L'essentiel des travaux, qui se sont intéressés au CCC, sont centrés sur les interactions conducteur/système et ont apporté des éléments de compréhension sur ses modalités d'utilisation et son efficacité. En revanche ils n'ont pas permis de rendre compte de façon suffisamment systématique de la manière dont les phénomènes sociaux participent à cet usage. L'objectif de notre article est ainsi de montrer en quoi l'usage et l'appropriation du régulateur de vitesse conventionnel ne sont pas le seul fait du conducteur et comment ils sont contraints par d'autres personnes, qu'il s'agisse des passagers de la voiture, d'autre usagers de la route ou encore de personnes non impliquées directement dans la situation de conduite.

\section{PRESENTATION}

Le régulateur de vitesse conventionnel est un système d'assistance à la conduite automobile dont la fonction est de conserver une allure prédéfinie par le conducteur, sans que ce dernier n'ait à maintenir un appui sur la pédale d'accélération. Deux formes d'instrumentation sont possibles : soit la commande prend la forme d'une manette placée derrière le volant, sur le même plan que l'indicateur de direction (figure 1), soit il s'agit de boutons situés directement sur le volant (figure 2). 


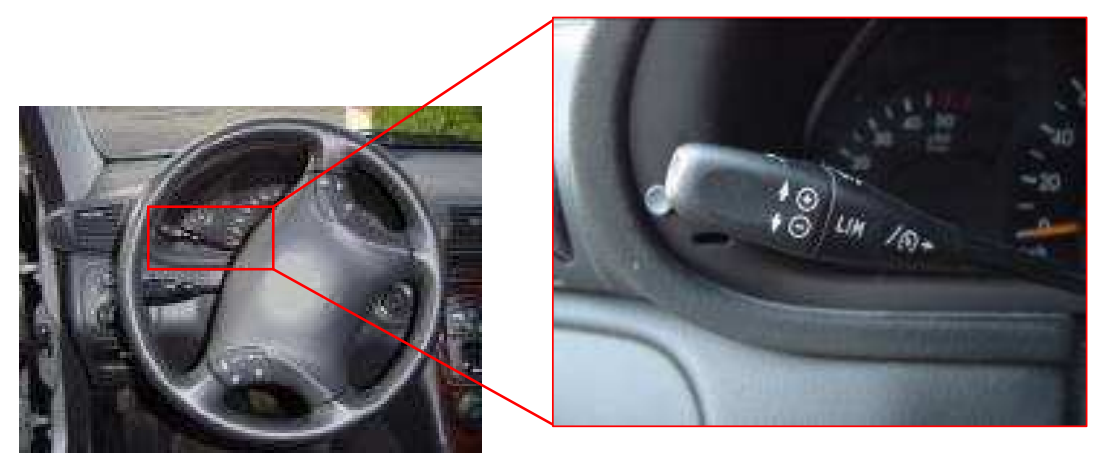

Figure 1. Instrumentation « manette » (Photos, contructeur allemand)

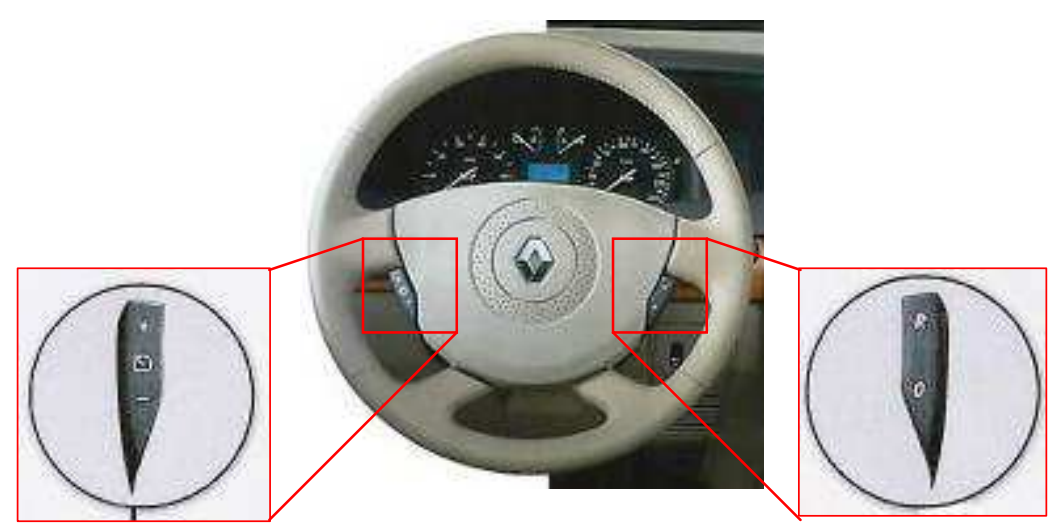

Figure 2. Instrumentation « au volant» (Document Renault, 2004)

Une séquence d'utilisation «typique » du régulateur de vitesse conventionnel se compose de trois phases : l'activation du dispositif, l'enregistrement de la vitesse de croisière désirée et la déconnexion du système. L'activation du dispositif se fait en actionnant l'interrupteur situé sur le tableau de bord (pour les instrumentations «manette » et « volant »). L'enregistrement de la vitesse de croisière désirée est réalisé à l'aide de la touche «+/- » pour l'instrumentation «volant» ou en actionnant vers le haut ou le bas la commande du système, pour l'instrumentation «manette », après avoir positionné le véhicule à l'allure souhaitée avec la pédale d'accélération. S'il le souhaite le conducteur peut faire varier la vitesse enregistrée en utilisant le même type de commande (incrémentation des pas de 1 ou $2 \mathrm{~km} / \mathrm{h}$, selon les modèles). Il est à noter qu'une allure précédemment enregistrée en mémoire peut être « rappelée » en appuyant sur le bouton «Resume », noté « R » dans la figure 2. Si la vitesse précédemment enregistrée est plus élevée que la vitesse courante, le véhicule accélère 
automatiquement. La déconnexion $d u$ système peut se faire en utilisant un des moyens suivants : appui sur la pédale de frein ou la pédale d'embrayage, positionnement du levier de vitesse au «point mort », enclenchement de l'interrupteur sur la position « Off », appui sur la

touche « $\mathrm{O} »$ (figure 2). Cette dernière touche permet de désactiver instantanément le dispositif mais la vitesse de croisière reste mémorisée dans l'ordinateur de bord. Lorsque le dispositif est activé, le conducteur peut dépasser la vitesse enregistrée en appuyant sur la pédale d'accélération. En relâchant la pédale, le régulateur de vitesse reprend le contrôle et la vitesse du véhicule se rétablit progressivement au niveau de l'allure de croisière prédéfinie.

\subsection{Les recherches portant sur l'usage du régulateur de vitesse et leur capacité à prendre en compte les phénomènes sociaux}

\subsubsection{Les principaux enseignements des travaux portant sur l'usage du CCC}

En mesurant la performance de conduite avec CCC et sans système d'assistance, les travaux portant sur le régulateur de vitesse conventionnel ont mis en évidence les avantages de ce mode de régulation au niveau de la sécurité (Warren, 1982 ; Smith, Ashby et Fairclough, 1994 ; Lheureux, Saad, Pianelli, Abric et Roland, 2006 ; Saad, Dionisio, Arslanian, Collaciuri, Legay, Vézier et Timianguel, 2006; Young et Reagan, 2007), du confort (Callahan, 1992 ; Lheureux et coll., 2006 ; Saad et coll., 2006 ; Young et Reagan, 2007) et de la consommation de carburant (Travis, 1982 ; Lheureux et coll., 2006).

Les points négatifs concernent principalement des difficultés liées à la gestion des interactions avec les autres usagers de la route non équipés de ce dispositif (Lheureux et coll., 2006), l'adoption de stratégies adaptatives de conduite présentant un risque relatif comme conduire uniquement avec «les mains », c'est-à-dire sans utiliser les pédales (Saad et coll., 2006), l'effort cognitif et physique lié à l'ajustement permanent de la vitesse de croisière aux conditions de circulation (Young et Reagan, 2007), le temps nécessaire pour s'approprier le 
système (Saad et coll., 2006 ; Young et Regan, 2007) et, enfin, des difficultés d'utilisation relatives à la mise en veille ou la désactivation du régulateur, lors de tentatives de rappel ou réactivation, ou en lien avec demauvaises attributions de fonction à une commande (Pagot, 2010).

Un autre corpus de recherche concerne les travaux utilisant l'activité de conduite avec CCC comme valeur de référence pour tester l'efficacité du dispositif nouvelle génération: le régulateur de distance («Adaptive Cruise Control» noté ACC). Couplé à un radar anticollision, l'ACC assure partiellement l'automatisation du contrôle de la distance intervéhicules et de la vitesse du véhicule (Bachmann, Naab, Reichart et Scrault, 2000). Les études comparant conduite $\mathrm{CCC} / \mathrm{ACC}$ montrent que le modèle conventionnel est moins confortable (Koziol, Inman, Carter, Robinson et Baker, 1999 ; Youngbin, 1997) et qu'il induit une charge de conduite plus importante ${ }^{1}$ (Stanton, Young et McCaulder, 1997). En matière de sécurité l'écart entre les deux modèles est moins flagrant : temps de réaction moins important avec CCC qu'avec ACC (Koziol et coll., 1999) mais distance inter-véhicules réduite (Fancher, Ervin, Sayer, Hagan, Bogard, Bareket, Mefford et Haugen, 1998) et vitesse moyenne plus élevée (Koziol et coll., 1999).

\subsubsection{Le décalage entre l'usage "solo» du régulateur de vitesse et l'aspect fortement « collectif» de l'activité de conduite}

Le niveau d'analyse de la majorité des travaux qui viennent d'être évoqués tend à être centré sur les interactions conducteur/dispositif, caractéristique des recherches sur les Interfaces Homme Machine (IHM). Ces études, même si elles ont apporté des éléments de connaissances précieux sur l'utilisation du régulateur de vitesse conventionnel, ont tendance à

\footnotetext{
${ }^{1}$ Même si l'hypothèse d'une activité supplémentaire de vigilance à l'égard du bon fonctionnement de l'ACC est avancée (Stanton et Young, 2005).
} 
privilégier l'idée d'un conducteur «solitaire » déconnecté de son environnement social et de la situation de conduite. Or il existe une littérature significative qui souligne l'importance de la dimension collective dans l'ensemble des activités de conduite. Les études distinguent une dimension collective dite «intravéhiculaire », caractérisant les phénomènes sociaux qui concernent des personnes présentes conjointement à l'intérieur de l'habitacle, d'une dimension «extravéhiculaire », désignant ces phénomènes lorsqu'ils concernent des personnes non présentes dans l'habitacle, et qui renvoie pour l'essentiel à ce que l'on désigne couramment en matière de prévention routière sous le terme de cohabitation entre les différents usagers de la route.

L'analyse de la littérature relative à la dimension collective intravéhiculaire, conduit à isoler trois grandes catégories de recherches : des études d'accidentologie, des études comportementales et des études sociologiques (voir Regan et Mitsopoulos (2003) pour une analyse plus détaillée). Les études d'accidentologie indiquent, dans leur majorité, que la présence des passagers a des conséquences sur la sécurité de la conduite. Ces travaux s'intéressent notamment à l'âge des personnes dans l'habitacle (Doherty, Andrey et McGregor, 1998), au nombre de passagers (Aldridge, Himmler, Aultman-Hall et Stamatiadis, 1999) et au sexe du conducteur (Chen, Baker, Braver et Li, 2000).

Les études comportementales montrent que les conducteurs ajustent leur conduite aux attentes des passagers (Baxter, et coll., 1990 ; Delhomme, 1994). Cette littérature, dans son ensemble, tend à considérer les passagers comme une source de distraction pour le conducteur (Zaidel, Paarlberg et Shinar, 1978 ; Wang, Knipling et Goodman, 1996) : l'influence des passagers sur la conduite portent essentiellement sur les répercussions négatives des conversations avec le conducteur (Goodman, Tijerina, Bents et Wierwille, 1999; Lee, Caven, Haake et Brown, 2001) et soulignent l'impact de la présence des passagers sur les comportements de conduite dans 90\% des cas (Rolls et Ingham, 1992). 
Certaines études d'orientation sociologique mettent en évidence un effet inverse, en soulignant la contribution significative des passagers comme aide pour le conducteur: l'orienter, répondre au téléphone à sa place, ajuster les dispositifs de confort, le maintenir en éveil, l'alerter d'un danger, ou commenter la vitesse pratiquée (Regan et Mitsopoulos, 2003). Les travaux disponibles relatifs à la dimension collective «extravéhiculaire » tendent à caractériser la conduite automobile comme une activité mobilisant plusieurs usagers de la route en interaction constante (Peretti-Watel, 2001). La conduite nécessite la mobilisation de « compétences sociales » qui permettent d'évoluer dans un environnement complexe en s'articulant avec les autres usagers. Les conducteurs sont également sensibles au comportement des autres usagers dans la détermination de leurs modalités de conduite : le choix de la vitesse du véhicule dépend de l'allure des véhicules présents dans l'espace proche et de la densité de circulation (Connolly et Aberg, 1993 ; Holland et Conner, 1996). L'essentiel de ces travaux indique que la conduite automobile est une «activité sociale » largement contrainte par le comportement d'autrui.

Nous venons de montrer ici d'une part, que la conduite automobile comme la plupart des activités humaines est faite d'interaction entre individus, le conducteur est influencé par le comportement des passagers et des autres usagers de la route, de l'autre, que l'essentiel des études portant sur le régulateur de vitesse conventionnel a tendance à considérer l'utilisation de ce dispositif comme le seul fait du conducteur. Ainsi, même si les travaux s'intéressant à la conduite avec CCC sont parvenus à fournir des éléments de précisions sur l'utilisation de ce système et à tester son efficacité, la littérature disponible ne permet pas de rendre compte de façon suffisamment précise de l'activité de conduite avec régulateur de vitesse telle qu'elle se déroule en contexte réel, c'est-à-dire dans un environnement qui intègre d'autres acteurs que le seul conducteur. 
1.1.3. L'intérêt de la prise en compte des travaux concernant l'ACC et de leur niveau d'analyse «élargi » au contexte social

Les études s'intéressant à la conduite avec ACC ont depuis quelques années montré l'implication d'autres individus que le conducteur dans l'usage de ce dispositif et souligné l'intérêt d'un niveau d'analyse «élargi » aux phénomènes sociaux. Par exemple, Saad et Villame (1996) et Fancher et coll. (1998) sont parvenus à démontrer que l'évolution du trafic conditionnait pour partie l'usage de l'ACC. Plusieurs travaux récents (par exemple Bjørkly, Jenssen, Moen et Vaa, 2003 ; Rudin-Brown et Parker, 2004 ; Ojeda et Nathan, 2006) ont réussi à observer la manière dont l'usage de l'ACC contraignait les modes d'interactions du conducteur avec les autres usagers. Saad et Malaterre (1982) ont pu montrer que les usagers de dispositifs régulant la vitesse de conduite étaient préoccupés par l'interprétation que pouvaient faire les autres conducteurs concernant leur comportement de conduite. Saad et coll. (2006) ont ainsi remarqué que les systèmes n'avaient pas seulement des effets sur le comportement du conducteur «assisté » mais également sur les perceptions et la conduite des autres conducteurs proches (dépourvus ou non du dispositif). Enfin, l'adoption d'un niveau analyse «élargi » a permis de décrire comment d'autres personnes que le conducteur intervenaient dans le processus d'apprentissage du système (comme les professionnels des concessions automobiles, Portouli et Papakostopoulos., 2006 ; Jenness, Lerner, Mazor, Osberg et Tefft, 2008). On peut d'ailleurs remarquer la préoccupation des futures campagnes européennes de tests des SAC et notamment de l'ACC (voir les projets FESTA, 2006-2008, et EuroFOT, 2008-2010) quant à la prise en compte des phénomènes sociaux autour du conducteur dans l'analyse de son comportement de conduite « assisté ». 
En conclusion, dans la poursuite des travaux les plus récents sur le CCC (Young et Regan, 2007 ; Lheureux et coll., 2006 ; Saad et coll., 2006 ; Pagot, 2010), qui commencent à envisager la nécessité d'élargir le champ d'analyse, et en tirant profit des recherches portant sur l'ACC, la présente étude tente de montrer en quoi la dimension collective et les jeux sociaux qui se déroule lors de la situation de conduite participent à l'usage du régulateur de vitesse. Cet article est une contribution à des questions qui restent pour l'instant sans réponse :

- Dans quelle mesure d'autres personnes que le conducteur sont-elles impliquées dans l'usage et l'appropriation du régulateur de vitesse conventionnel ?

- Comment l'activité des différents acteurs participant à la situation de conduite contraint-t-elle l'usage du régulateur de vitesse?

- Jusqu'à quel point l'usage du CCC est-il source de satisfaction/gêne, sécurité/risque pour les autres personnes de la situation de conduite à l'intérieur et à l'extérieur de l'habitacle?

\subsection{Cadre théorique : une approche de l'usage du régulateur de vitesse en situation réelle qui intègre les phénomènes sociaux}

Le cadre théorique de cette étude s'inspire des préconisations de Villame et Theureau (2001) et Ciaccabue et Saad (2008) concernant les apports des théories de «l'action située » (Suchman, 1987 ; Theureau, 2004) à la conception des systèmes d'assistance à la conduite automobile. Les théories de «l'action située » se situent au carrefour de plusieurs disciplines : anthropologie cognitive, psychologie, microsociologie, et ont pour but d'explorer les relations entre le contexte, la cognition et l'action. Cette perspective donne une place centrale à l'activité humaine, la considérant comme une totalité complexe intégrant émotions, attentions, perceptions, actions, communications, interprétations. Au lieu de réduire l'usage du régulateur à la juxtaposition d'un certain nombre de variables (temps de réaction, attention, charge 
mentale, distance inter-véhicules...), l'ambition est de prendre en compte les phénomènes dans leur complexité en milieu «naturel » de conduite, c'est-à-dire pris dans ses circonstances sociales, culturelles et techniques complexes (Theureau, 2004). Ce choix méthodologique reflète l'idée forte que l'usage du CCC est étroitement lié au contexte dans lequel il se déroule. Dit autrement, le conducteur interagit à chaque instant du déroulement de la conduite avec son environnement social (passagers, piétons, motocyclistes, autres conducteurs...) et son environnement matériel (configuration de la route, panneaux, interface du CCC...), et de cette interaction résulte un comportement singulier et particulier à ce moment de l'action.

Le niveau d'analyse de ce type d'approche «située» offre la possibilité de considérer l'activité de conduite à un niveau individuel-collectif, dans la mesure où d'autres personnes contraignent par épisodes cette activité, ce qui fait que l'activité individuelle est en fait individuelle collective. Concrètement, au cours de l'usage du CCC s'articulent des périodes d'activité individuelle, où le conducteur est strictement mis en jeu, et des périodes d'activités collectives, où interviennent les passagers et les autres usagers de la route, pour autant que ces derniers jouent un rôle significatif pour le conducteur. Une partie de ces idées est notamment développée par Hutchins (1995) dans un programme de recherche en sciences du travail autour de la cognition distribuée qui montre comment la prise en compte du groupe culturellement constitué (intégrant l'environnement social et matériel) plutôt que de la cognition individuelle comme d'unité d'analyse permet d'apporter des éléments de connaissances de l'activité humaine. L'adoption de ce niveau «individuel-collectif » pour l'étude des comportements de conduite avec assistance fait suite aux conclusions de Saad, Hjälmdahl, Cañas, Alonso, Garayo, Macchi, Nathan, Ojeda, Papakostopoulos, Panou et Bekiaris (2004) au sein du projet $\operatorname{AIDE}^{2}$ (2004-2008) préconisant la prise en compte d'une

\footnotetext{
${ }^{2}$ Le projet européen AIDE (2004-2008, 6ème PCRD) a pour objectif de développer des méthodologies et des interfaces homme/machine destinés à l'intégration de dispositifs technologiques dans l'automobile.
} 
approche «multi-niveaux » de l'usage des SAC qui permette d'étudier de manière conjointe l'activité du conducteur considéré et ses interactions avec les autres usagers de la route. Cette ligne de recherche a émergé du manque de travaux empiriques intégrant d'autres individus que le conducteur «assisté ». Cette approche préconisée par Saad et coll. (2004) et déjà testée dans Saad et coll. (2006) et Lheureux et coll. (2006) nécessite d'être développée de manière plus approfondie. Au niveau méthodologique, le recueil de données nécessite ainsi de prendre davantage en compte les collaborations entre l'ensemble des individus participant à l'action et la façon dont ils contraignent/facilitent l'activité du conducteur. Ce niveau d'analyse permet d'étudier conjointement les articulations individu-collectif en évitant deux écueils : celui de « diluer» le conducteur dans le groupe ou à l'inverse de l'isoler de son environnement social.

\section{METHODES}

Les méthodes de recueil et d'analyse de données utilisées dans la présente étude font partie de l'observatoire du cours d'action (Theureau, 2004) destiné à la description de l'activité humaine en situation réelle. Cet observatoire, qui constitue un ensemble flexible et ouvert de principes, peut être adapté aux caractéristiques spécifiques de l'activité de conduite automobile et des questions de recherche posées.

\subsection{Participants}

Dix conducteurs français (8 hommes et 2 femmes) âgés de 25 à 65 ans ont été recrutés par contacts externes pour cette étude. Tous possèdent un régulateur de vitesse conventionnel sur leur véhicule personnel (tableau 1). Des pseudonymes ont été utilisés afin de garantir l'anonymat des participants. 


\begin{tabular}{|c|c|c|}
\hline Nom & Âge & Profil \\
\hline André & 65 ans & $\begin{array}{l}\text { Expérience avec } C C C: 5^{\text {ème }} \text { voiture consécutive munie de CCC } \\
\text { Habitude d'utilisation du CCC : usage fréquent sur grandes routes dégagées } \\
\text { Profession/signes particuliers : retraité }\end{array}$ \\
\hline Christophe & 35 ans & $\begin{array}{l}\text { Expérience avec CCC : } 3^{\text {ème }} \text { voiture consécutive munie de CCC } \\
\text { Habitude d'utilisation du CCC : usage fréquent de } 1995 \text { à } 2005 \text { puis aucune utilisation } \\
\text { Profession/signes particuliers : directeur d'entreprise }\end{array}$ \\
\hline Denis & 42 ans & $\begin{array}{l}\text { Expérience avec CCC : } 2^{\text {ème }} \text { voiture consécutive munie de CCC } \\
\text { Habitude d'utilisation du CCC : usage systématique lors de tous les trajets } \\
\text { Profession/signes particuliers : informaticien }\end{array}$ \\
\hline Jérôme & 25 ans & $\begin{array}{l}\text { Expérience avec CCC : } 1 \text { ère voiture munie de CCC } \\
\text { Habitude d'utilisation du CCC : usage peu fréquent (autoroute vide) } \\
\text { Profession/signes particuliers : étudiant }\end{array}$ \\
\hline Michel & 56 ans & $\begin{array}{l}\text { Expérience avec CCC : } 4^{\text {ème }} \text { voiture consécutive munie de CCC } \\
\text { Habitude d'utilisation du CCC : n'a jamais utilisé de CCC } \\
\text { Profession/signes particuliers : agent administratif / conjoint de Stéphanie / peu } \\
\text { familier avec les dispositifs technologiques de la vie courante }\end{array}$ \\
\hline Pierre & 32 ans & $\begin{array}{l}\text { Expérience avec CCC : } 1^{\text {ère }} \text { voiture munie de CCC / essai du CCC en } 1992 \text { sur un } \\
\text { camping car de location aux Etats-Unis } \\
\text { Habitude d'utilisation du CCC : usage peu fréquent (long trajet sur autoroute) } \\
\text { Profession/signes particuliers : enseignant }\end{array}$ \\
\hline Sarah & 27 ans & $\begin{array}{l}\text { Expérience avec CCC }: 1^{\text {ère }} \text { voiture munie de CCC } \\
\text { Habitude d'utilisation du CCC : usage très rare } \\
\text { Profession/signes particuliers : kinésithérapeute }\end{array}$ \\
\hline Louis & 47 ans & $\begin{array}{l}\text { Expérience avec CCC : } 1 \text { ère voiture munie de CCC } \\
\text { Habitude d'utilisation du CCC : usage fréquent sur grandes routes dégagées } \\
\text { Profession/signes particuliers : sans profession }\end{array}$ \\
\hline Jean & 38 ans & $\begin{array}{l}\text { Expérience avec CCC }: 4^{\text {ème }} \text { voiture personnelle consécutive munie de CCC / utilise } \\
\text { depuis } 5 \text { ans les voitures de sa société munies de CCC } \\
\text { Habitude d'utilisation du CCC }: \text { usage fréquent sur autoroutes } \\
\text { Profession/signes particuliers : commercial }\end{array}$ \\
\hline Stéphanie & 53 ans & $\begin{array}{l}\text { Expérience avec CCC }: 1^{\text {ère }} \text { voiture munie de } \mathrm{CCC} / \text { utilise la voiture de Michel } \\
\text { Habitude d'utilisation du CCC }: \text { usage très rare (autoroute vide) } \\
\text { Profession/signes particuliers }: \text { infirmière / conjointe de Michel }\end{array}$ \\
\hline
\end{tabular}

Tableau 1. Détail des participants et profil d'usage du régulateur de vitesse conventionnel

\subsection{Procédure}

\subsubsection{Recueil de données}

Le recueil des données s'est étalé sur une période de six mois (septembre 2007-février 2008).

Chaque conducteur a été suivi pendant une semaine au cours de ses trajets quotidiens avec sa

propre voiture. Les données sont recueillies avant, pendant et après cette semaine de conduite

(appelée « session de conduite » dans la suite) (tableau 2). 


\begin{tabular}{llll}
\hline $\begin{array}{l}\text { Période de recueil } \\
\text { de données }\end{array}$ & $\begin{array}{l}\text { Avant la session } \\
\text { de conduite }\end{array}$ & $\begin{array}{l}\text { Pendant la session de } \\
\text { conduite }\end{array}$ & $\begin{array}{l}\text { Après la session de } \\
\text { conduite }\end{array}$ \\
\hline \multirow{2}{*}{$\begin{array}{l}\text { Méthodes } \\
\text { utilisées }\end{array}$} & $\begin{array}{l}\text { Entretien } \\
\text { informel }\end{array}$ & $\begin{array}{l}\text { - Observations } \\
\text { systématiques }\end{array}$ & $\begin{array}{l}\text { - Méthode } \\
\text { biographique }\end{array}$ \\
& & $\begin{array}{l}\text { - Verbalisations } \\
\text { interruptives }\end{array}$ & $\begin{array}{l}\text { - Entretien } \\
\text { d'explicitation }\end{array}$ \\
\hline
\end{tabular}

Tableau 2. Déroulement du recueil de données et méthodes utilisées

Avant la session conduite, ont été recueillies des données sur le profil des conducteurs (données démographiques, style de conduite, habitude d'usage du CCC, familiarité avec les systèmes technologiques) et les caractéristiques de leur culture (l'ensemble des éléments de généralité issus de l'expérience passée du conducteur) au moyen d'un entretien informel.

Au cours de la session de conduite, des observations ont été menées de manière continue lors de la totalité des trajets habituels des participants. Le chercheur, occupant le siège passager, notait sur un carnet ce qui dans l'activité du conducteur concernait le CCC (modalités et situations d'activation/désactivation, comportement de conduite avec CCC, commentaires, difficultés observées...). Le chercheur observait et notait également les comportements (conduite/communications verbales et/ou gestuelles) des autres acteurs de la situation (passagers/autres usagers) qui pourraient être liés à la conduite avec $\mathrm{CCC}$ du conducteur étudié. En complément de ces observations, des verbalisations de type «interruptives » (Theureau et Jeffroy, 1994) ont été recueillies pendant la conduite. Le chercheur demandait au conducteur d'expliciter ses actions par des questions qui font strictement référence à ce qui a été fait et qui n'induisent pas de généralisations. Ces questions sont posées à des moments où elles troublent le moins possible le déroulement normal de la conduite.

Après la session de conduite, un entretien d'explicitation (Vermersch, 1990) s'appuyant sur la description de la «ligne d'usage du régulateur de vitesse » complétée par le conducteur a été mené avec chaque participant. L'emploi de la «méthode biographique » à travers la conception de la « ligne de vie » a pour objectif d'identifier les événements vécus significatifs du point de vue du participant tout au long de sa vie. Dans le cadre professionnel, la « ligne de 
vie professionnelle» Mevel (2000) consiste à ce que l'opérateur reconstruise lui-même graphiquement son récit de vie professionnelle. Nous avons détourné cette méthode de son contexte d'origine pour en faire une «ligne d'usage du régulateur de vitesse ». De manière concrète, on demande au conducteur de dessiner un axe des temps horizontal où la situation présente de l'entretien est représentée tout à droite de l'axe. Le conducteur choisit alors l'échelle de temps souhaitée, puis identifie et repère sur l'axe les moments clés, les principales étapes, les situations déterminantes de son histoire d'usage du système. La méthode de la biographie n'est pas une autobiographie parce que le chercheur demande à ses interlocuteurs de reconstruire leurs parcours de vie selon une direction qu'il considère, lui, comme décisive par rapport à sa recherche (Dominicé, 2002). Cette technique de recueil de données permet (a) d'avoir une vision d'ensemble de la dynamique d'usage du CCC du conducteur qui peut servir de point de repères au chercheur et au participant lors du passage à la verbalisation, (b) d'identifier les épisodes clés qui se sont déroulés avant l'entretien «ici et maintenant » et qui touchent notamment au processus d'appropriation du système, (c) de réaliser « une entrée en matière » dans l'entretien avant de passer à la verbalisation qui peut être perçue comme trop «impliquante ».

S'appuyant sur cette ligne d'usage complétée, un entretien d'explicitation (Vermersch, 1990) d'une durée approximative de 45 minutes a été mené avec chaque participant. L'entretien d'explicitation est une technique d'aide à la verbalisation des éléments implicites de l'action, en relation à une situation spécifiée, ayant pour avantage de quitter le registre des généralités, pour aller au plus près des expériences subjectives. Le chercheur s'attache à poser des questions strictement en référence à ce qui a été fait et dit, afin que l'acteur puisse se replacer le plus possible dans le contexte dynamique de la situation vécue. Dans notre étude, l'entretien d'explicitation a été utilisé pour préciser les moments significatifs de l'histoire d'usage du CCC des conducteurs qu'ils ont eux-mêmes identifiés sur la ligne. Il s'agit de 
replacer le conducteur dans le contexte de l'événement considéré et de l'aider à décrire ses actions, ses sensations, ses émotions, les situations telles qu'elles étaient et le comportement des autres personnes qui participaient à l'action. Nous avons pris la décision à travers cette technique de décrire les situations importantes de l'appropriation et de l'usage du CCC pour les conducteurs étudiés et ensuite de repérer dans quelle mesure ces situations comportaient une ou des dimensions collectives. Cette approche « située » permet notamment de conserver le contexte d'occurrence de l'événement «collectif » considéré et d'apprécier le poids de cet événement et de la participation de tous les acteurs dans la dynamique d'usage du système.

\subsubsection{Mise en forme et analyse des données}

Mise en forme. Les données recueillies avant la session de conduite ont été laissées tel quel. Elles servent à fournir des données stables sur l'état et la culture des participants et par exemple à définir les profils d'utilisateurs repérés dans le tableau 1. Les données récoltées pendant la session de conduite, une fois retranscrite verbatim, ont été mises en forme dans des protocoles verbaux à deux volets: un premier volet où sont placées les données d'observations et un second volet où sont répertoriés les verbalisations interruptives. Les données issues des lignes d'usage sont laissées tel quel. Elles fournissent déjà des données brutes exploitables et servent surtout de support pour la réalisation des entretiens. Les données d'entretiens d'explicitation ont été retranscrite verbatim. Chaque situation de conduite identifiée sur la ligne et précisé par le conducteur à été distinguée et numérotée.

Analyse. L'analyse comporte trois étapes. La première consiste à répertorier l'ensemble des situations de conduite avec CCC (de la session de conduite observée et de l'ensemble de l'histoire d'usage) qui comportent une dimension collective (codage réalisé par les deux chercheurs à l'aveugle). Lors de la deuxième étape, les catégories d'analyse ont été construites sur le modèle «top-down » (raisonnement déductif « classique ») et «bottom-up » 
(raisonnement inductif), c'est-à-dire que les catégories émergent pour une part des questions de recherches et pour une autre des premières données collectées. L'immersion dans les données empiriques sert de point de départ au développement des catégories d'analyse et permet de conserver le lien d'évidence avec les données de terrain. Cette démarche inductive initiée par Glaser et Strauss (1967), sous le nom “Grounded Theory”, permet de générer de manière systématique des catégories d'analyse à partir des données récoltées tout au long de la recherche. L'ensemble des données a été codé lors de la troisième étape avec les catégories d'analyse identifiées.

\section{RESULTATS}

Les résultats majeurs de cette étude montrent : (1) les effets de la présence de passagers non habitués à une vitesse régulée sur la conduite, (2) la façon dont l'usage du régulateur de vitesse peut être envisagé comme le jeu d'interaction sociales, (3) les difficultés de régulation de la vitesse dans le trafic entre véhicules équipés et non équipés, (4) les modifications de distance inter-véhicules et leurs conséquences sur la cohabitation entre automobilistes et (5) l'existence d'une dimension collective forte dans le processus d'appropriation du système.

\subsection{Les passagers non habitués à une vitesse régulée sont une contrainte pour le conducteur}

L'utilisation du régulateur de vitesse génère une conduite à allure plus régulière (c'est-à-dire. moins d'interventions sur la commande du régulateur de vitesse que d'appuis sur les pédales lors d'une conduite classique) et donc une adaptation moins fine à la configuration de la route empruntée. Or, les données montrent que cette régularité constitue une gêne pour les passagers et spécialement pour l'individu situé sur le siège à côté du conducteur (pour cinq 
des dix sujets). D'après les participants, les passagers prétendent fréquemment que l'allure de la voiture est trop élevée et non adaptée à la situation. Les conducteurs déclarent pourtant mémoriser une vitesse égale voire en dessous de l'allure réglementaire :

«Ma femme me dit: " attention tu roules trop vite!" Alors que je ne roule pas plus vite que d'habitude. Elle a l'impression que j'aborde les virages trop vite parce que, pour elle, l'allure n'est pas adaptée à la route, alors que je suis en dessous de la vitesse réglementaire, elle me dit qu'elle est gênée quand je conduis avec régulateur.» (Pierre, verbalisations interruptives)

«Presque à chaque fois que je conduis avec régulateur, mon épouse, qui est sur le siège passager, me dit que je conduis comme " une patate!" Je ne roule pas vite pourtant!»(Denis, Verbalisation concomitante)

On peut penser que les habitudes corporelles des passagers construites au cours de leurs expériences antérieures (conducteur et/ou passager) sont liées à une conduite à allure irrégulière, faite d'accélérations, de décélérations, de freinages.... Les propos recueillis auprès de Denis illustrent ce décalage :

«Normalement, sans régulateur, admettons que c'est limité à $90 \mathrm{~km} / \mathrm{h}$, on va rouler à $95 \mathrm{~km} / \mathrm{h}$, et puis dans un virage on va descendre à $85 \mathrm{~km} / \mathrm{h}$, et puis on va accélérer en sortie de virage. Tout le monde est habitué et principalement les passagers à ce genre de conduite. Alors qu'avec le régulateur tu peux très bien rouler sur cette même route en gardant une vitesse constante et ça, ça gêne les passagers! (Denis, verbalisations concomitantes)

L'absence d'adéquation entre les sensations vécues par les passagers au cours de la conduite avec régulateur de vitesse et celles de leurs expériences antérieures fait que la conduite avec ce dispositif les met en difficulté. Les plaintes des passagers ne semblent pas être des événements isolés :

«Au début, ma fille m’a fait des remarques sur la conduite.A chaque fois que je vais rouler avec régulateur de vitesse, les passagers vont trouver que je roule 
trop vite et ils me le font remarquer! Donc systématiquement, je fais très

attention à ma conduite dès que j'enclenche le régulateur et qu'il y a des passagers dans ma voiture ». (Jérôme, entretien d'explicitation)

Au bilan, les résultats indiquent que dans l'optique de préserver le confort des individus présents dans leur véhicule, les conducteurs étudiés sont parfois amenés à désactiver le dispositif. Cela montre (1) que les critiques des passagers induisent de profonds changements dans les modalités habituelles d'usage du régulateur de vitesse et (2) que les conducteurs apportent un «soin» au confort de leur(s) passager(s), même en l'absence de plaintes explicites des ces derniers. Le confort des passagers dans la voiture est une réelle préoccupation pour les conducteurs.

Dans un ordre autre idée, il peut être intéressant de remarquer que l'élément perçu par les passagers est un décalage d'allure. On peut penser que leurs expériences de conduite à vitesse non régulée sont trop divergentes d'une conduite à vitesse régulée. On peut même faire l'hypothèse de l'existence d'un processus d'apprentissage d'être passager d'un véhicule conduit à vitesse régulée.

\subsection{Le régulateur de vitesse comme instrument privilégié de reproduction des jeux sociaux habituels au sein de l'habitacle}

Les données présentées dans les extraits suivants montrent comment l'outil « régulateur de vitesse » matérialise dans l'habitacle de l'automobile le type de relation sociale qu'entretiennent les personnes dans la vie quotidienne. Le premier extrait concerne les rapports entre deux conjoints Stéphanie et Michel, qui conduisent la même voiture. L'entretien informel réalisé avec Michel a montré qu'il n'utilisait pas le régulateur de vitesse présent sur son véhicule. Lors de l'achat de la voiture, le vendeur lui aurait conseillé de ne pas activer ce système pour des raisons de sécurité. Il interdit ainsi formellement à sa conjointe Stéphanie d'utiliser ce système. Ce contexte particulier a permis d'envisager Michel non plus 
uniquement comme conducteur, mais comme passager, et donc d'étudier finement la relation entre Michel et Stéphanie. Deux situations peuvent être distinguées selon que Michel et Stéphanie sont présents dans la voiture ou que Stéphanie est seule au volant. Les données indiquent que Stéphanie respecte dans la majorité des cas les recommandations de son mari concernant l'usage du CCC quand celui-ci se situe sur le siège passager, mais qu'elle se risque parfois à des essais sans sa permission :

«Michel m'a strictement défendu de toucher au régulateur de vitesse. Quand il est à côté de moi, j'essaye quand même d'utiliser le régulateur pour voir ce que ça fait. J'active le système très furtivement, de temps en temps, mais Michel risque de m'engueuler donc je l'enlève rapidement. (...) Michel me fiche la trouille. Quelque fois j'ai un peu de mal à mettre le régulateur, je cherche, je bidouille. Oui je mets le régulateur quand il est à côté, sans lui dire, mais je stresse quand même.

Vous mettez le régulateur, même quand il est à côté de vous?

Oui. Il est sur le siège passager, je fais des essais, je ne lui dis pas mais je l'enlève vite! Je stresse. »(Stéphanie, données entretien)

Les observations montrent que Stéphanie manipule discrètement la manette du régulateur de vitesse située sur la gauche du volant de la main gauche. Elle prétend activer le système quelques secondes, "juste le temps de voir comment ça marche », uniquement au cours de trajets sur autoroutes dégagées. Après ces brèves tentatives, elle désactive le régulateur de vitesse pour que Michel ne se rende pas compte que le système est enclenché. Les échanges avec ce dernier montrent qu'il est persuadé que sa femme n'utilise jamais le dispositif en sa présence.

Dans le cas où Stéphanie se trouve seule au volant du véhicule, on aurait pu faire l'hypothèse d'une utilisation plus poussée du CCC. Cependant les données montrent le contraire : 
«Même quand Michel n'est pas là, je panique en pensant à lui, s'il voit que j'ai utilisé son régulateur de vitesse !! En fait, je me rends compte que j’ai du mal à utiliser le régulateur, même quand Michel n'est pas dans la voiture avec moi. » (Stéphanie, données d'entretien)

En l'absence de Michel, Stéphanie n'utilise pas plus fréquemment ni plus longuement le régulateur de vitesse. Elle tente de comprendre son fonctionnement par des essais brefs sur autoroute. Elle prétend désactiver le dispositif et paniquer en repensant aux mises en garde de son mari. Cet exemple illustre bien comment le CCC sert le conflit dans le couple à l'intérieur de l'habitacle de la voiture et peut être considéré comme un prolongement du fonctionnement habituel du couple dans la vie quotidienne.

Le jeu dont il s'agit maintenant est un peu différent et concerne un défi entre collègues de travail. Jean est membre d'un groupe de commerciaux dont l'entreprise est basée en région Centre et qui effectue fréquemment des trajets pour se rendre dans une filiale localisée à Marseille. Dans l'extrait suivant, Jean nous explique le jeu qu'il a instauré avec ses collègues :

«A chaque fois qu'un commercial descend à Marseille, il enclenche le régulateur de vitesse et il compte le nombre de fois qu'il appuie sur la pédale de frein. L'objectif c'est d'appuyer le minimum de fois sur le frein durant tout le parcours. Et moi, à ce jeu, je suis super fort, une fois je suis descendu à Marseille sans toucher une seule fois à la pédale de frein! Et ça fait quand même 700 bornes! »(Jean, données d'entretien)

Les commerciaux cités ne font que reproduire les compétitions auxquelles ils se livrent dans leurs relations amicales et dans leurs activités de travail quotidiennes. 
Dans un autre ordre d'idée, l'attitude de Louis vis-à-vis des passagers qu'il transporte est caractéristique de la relation qu'il entretien avec autrui dans la vie quotidienne. Lorsque Louis active le régulateur de vitesse et passe en mode de conduite à allure régulée, ça se voit ou plutôt les passagers le remarquent. Louis ne touche plus le volant des doigts mais l'effleure des cuisses, place ses mains derrière la tête, croise ses jambes sous le siège et discute avec désinvolture avec le ou les passagers présents dans la voiture. Des éléments de compréhension de ce comportement ostentatoire sont avancés par l'intéressé :

«J'aime bien qu'on remarque que je suis tranquille au volant, que je ne peine pas, que c'est facile. C'est pour ça que je montre que je suis aussi relâché avec les mains derrières la tête et les jambes sous le siège, surtout si c'est une nana la passagère (rire)! Mais attention je ne fais pas ça dans n’importe quelle situation! Je le fais uniquement sur autoroute et quand le trafic n'est pas trop dense. » (Louis, données d'entretien)

On voit bien ici que l'outil régulateur de vitesse est instrumenté pour servir un type de comportement de valorisation du conducteur à l'égard des autres passagers. Comportement que Louis ne réserve pas à la conduite mais qu'il adopte également dans la vie quotidienne. Au bilan, les trois situations présentées permettent d'apprécier comment le régulateur de vitesse devient un outil qui s'inscrit dans un régime habituel d'interactions auxquelles se livrent les individus au sein de leur véhicule que ce soit dans des conditions de travail ou dans la vie quotidienne.

\subsection{Les difficultés de régulation de la vitesse dans le trafic entre véhicules équipés et non équipés}

D’après les données recueillies, ce n'est pas la régularité de l'allure proprement dite qui pose des problèmes aux conducteurs, mais la cohabitation avec les autres usagers de la route dont les véhicules sont dépourvus de régulateur de vitesse : 
"C'est énervant, tu roules à côté de mecs qui vont une fois à 142, une fois à 138 $\mathrm{km} / \mathrm{h} .$. Le gars il me double, après il ralenti donc suis obligé de ralentir et de le doubler, après il va accélérer et me doubler encore une fois. Ça m'arrive très souvent sur l'autoroute. »(Denis, verbalisations concomitantes)

«Je suis très sensible à l'allure des autres automobilistes quand je conduis avec régulateur. Je vois bien les gens qui n'ont pas le régulateur, ils roulent à une vitesse irrégulière, même si en moyenne ils roulent à la même vitesse que moi. Mais c'est super énervant parce qu'ils te gênent sans arrêt !» (Jérôme, verbalisations concomitantes)

«Sur l'autoroute, les autres voitures sont embêtantes: tu es obligé d'accélérer pour doubler parce qu'elle ralenti et après elle te double et ainsi de suite. Moi je veux être tranquille, pénard avec mon régulateur!» (Pierre, verbalisations concomitantes)

La gêne occasionnée semble être générée par l'interaction proche entre le véhicule considéré, évoluant à allure régulière (avec $\mathrm{CCC}$ ), et un autre véhicule roulant à allure plus irrégulière (sans CCC). Le comportement de ce dernier contraint l'utilisateur de régulateur de vitesse, d'une part, à faire preuve de plus d'attention qu'il ne souhaite à l'égard du trafic, et d'autre part, à adopter des comportements particuliers :

«Dans ce cas là, j'accélère un bon coup sur un kilomètre pour distancer la voiture de 500 mètres. Je roule à 20 ou $30 \mathrm{~km} / \mathrm{h}$ de plus, sur une centaine de mètres et ça suffit pour ne plus entendre parler du conducteur gênant. » (Louis, verbalisations concomitantes)

"Quand un mec ralentit systématiquement dans les côtes et qu'après il me double sur le plat, ça m’énerve! Dans la majorité des cas, je finis par le doubler par la droite. Je reste dans ma file, à mon allure, alors que lui est sur la voie du milieu. C'est un peu dangereux, je sais, mais je n'ai pas envie de désactiver le 
régulateur ou de faire l'effort d'aller complètement sur la voie de gauche pour le doubler. » (André, entretien d'explicitation)

Des manœuvres relativement risquées sont donc mises en œuvre par les conducteurs observés pour rompre l'interaction directe avec le véhicule «gênant»: soit des accélérations brutales au-delà de la vitesse autorisée (pour Denis et Jean), soit des dépassements par la droite sur autoroute (pour André, Pierre et Jérôme). L'utilisateur reprend ensuite sa conduite à allure régulée.

En résumé, la conduite associée à l'usage du régulateur de vitesse est une conduite asservie au système, fonctionnant sur un régime automatique, sans effort significatif pour le conducteur. Elle diffère de la conduite sans dispositif, faite d'accélérations et de décélérations. Ce décalage occasionne plusieurs types de difficultés pour le conducteur qui adopte des manœuvres particulières pour réduire cette gêne.

\subsection{Les modifications de distance inter-véhicules et leurs conséquences sur la cohabitation entre automobilistes}

L'objectif de la majorité des conducteurs étudiés étant «d'en faire le moins possible au volant », le régulateur de vitesse constitue un système d'assistance intéressant, car il permet de diminuer l'activité au niveau des membres inférieurs. Or les données montrent qu'en situation de conduite réelle, il est nécessaire d'activer et de désactiver fréquemment le dispositif en fonction de l'évolution de la densité du trafic rencontré. On peut citer les exemples de Pierre et de Denis :

\footnotetext{
"C'est super casse pied de désactiver le régulateur, de réaccélérer, le réenclencher tout le temps. »(Pierre, notes d'observation)

«Là je suis sur autoroute, je me mets à 140, t’as envie de rien faire, mais il y a d'autres voitures sur l'autoroute et je n'ai pas envie de déconnecter le
} 
régulateur et puis de le remettre sans cesse.» (Louis, verbalisations concomitantes)

L'effort physique et cognitif demandé au conducteur ne semble pas être aussi allégé que prévu avec l'activation du régulateur de vitesse. Au contraire, les données laissent à penser que le conducteur doit, en plus des actions de conduite habituelles, manipuler et faire preuve d'attention à l'égard du dispositif. Dans l'optique de minimiser leurs efforts, les conducteurs observés décident de différer dans le temps, au maximum, la désactivation du système. En d'autres termes, ils laissent le régulateur de vitesse le plus longtemps possible enclenché, même dans des conditions de trafic qui deviennent denses :

«Moi j'avoue qu'à chaque fois que le régulateur est en marche, j'attends le dernier moment pour l'enlever. Et c'est là que j'ai tendance à me rapprocher vachement du véhicule précédent, pas pour le pousser au cul, mais pour dire “ok ça passe” sans toucher au régulateur. » (André, entretien d'explicitation) «Ca m'énerve de déconnecter le régulateur à chaque fois que j’ai une voiture devant moi donc je le désactive au dernier moment. Je fais ça tout le temps, sur nationale ou sur autoroute, sinon le système ne sert plus à rien, tu le manipules toutes les trente secondes! »(Denis, entretien d'explicitation)

Ces stratégies d'adaptation semblent réduire la distance de sécurité entre véhicules et provoquer des conflits avec le conducteur qui précède :

«Il m'arrive souvent de me faire engueuler par d'autres automobilistes parce qu'ils croient que je veux leur rentrer dedans. Les mecs ne comprennent pas, ils me voient approcher et ne pas ralentir. Mais c'est que je ne veux pas m'embêter à enlever le régulateur à chaque fois. »(Jean, verbalisations concomitantes).

"C'est vrai avec le régulateur j'ai tendance à coller vachement la voiture devant moi, je m'en rends compte! J'ai même failli avoir plusieurs accidents tellement j'étais prêt de l'autre voiture, souvent c'est passé très très juste! » (Louis, verbalisations concomitantes). 
Les résultats témoignent donc d'un décalage entre d'un côté, l'économie de moyens et l'utilité annoncée du système en termes de confort, et d'un autre, l'utilisation du dispositif en conditions réelles de trafic, relativement coûteuse en termes d'énergie. Ce décalage amène les conducteurs à développer des stratégies « conservatrices » d'usage du système, afin de réduire l'effort de conduite avec régulateur de vitesse. Des stratégies qui peuvent présenter un risque relatif.

\subsection{L'existence d'une dimension collective forte dans le processus d'appropriation du système}

\subsubsection{L'entourage du conducteur comme composante majeure de l'appropriation du $C C C$}

De manière intuitive, on peut supposer que pour utiliser de manière efficace le CCC un minimum de connaissances doit être requis : des connaissances liées à la manipulation (comment activer/désactiver le système ? Sur quel bouton appuyer pour enregistrer la vitesse courante ?...) et aux conditions d'utilisation de l'outil (sur quel type de route utiliser préférentiellement le dispositif ?...). De manière plus approfondie, les données montrent que ces connaissances résultent pour tous les participants d'un processus collectif. Les «formateurs » sont des collègues de travail du conducteur, font partie de sa famille (conjoint, enfants, parents) ou appartiennent à la sphère amicale. Trois participants prétendent que des membres de leur environnement de travail ont contribué à leur apprendre le «bon usage »du régulateur de vitesse. Pour Jérôme, il s'agit de son patron :

"C'est mon patron qui m'a appris à me servir du régulateur de vitesse. Il est monté dans la voiture, sur le siège passager, et il m'a dit de démarrer. On est parti sur l'autoroute, il n'y avait pas beaucoup de trafic, il m'a demandé d'accélérer jusqu'à $130 \mathrm{~km} / \mathrm{h}$ et c'est à ce moment qu'il m'a montré comment on pouvait enregistrer la vitesse, désactiver le système, rappeler la vitesse... Il y a 
avait quand même des voitures à côté de nous. J'ai trouvé ça un peu dangereux! »(Jérôme, entretien d'explicitation)

Les «formateurs » peuvent également prendre la forme de personnes de l'entourage familial des conducteurs. C'est le cas pour la majorité des participants (cinq sur dix) dont Christophe :

"J'ai découvert le régulateur de vitesse grâce à mon père. Il venait d'acheter une voiture qui en était équipée. Il m'a tout de suite montré le système et son intérêt. J'ai trouvé ça génial! Ensuite il m'a rapidement expliqué comment on faisait pour l'utiliser et à quoi servaient les commandes. Ça m’a paru très simple et c'est d'ailleurs la seule explication que j'ai eue jusqu'à maintenant ! » (Christophe, entretien d'explicitation

Pour deux autres conducteurs, ce sont cette fois des personnes appartenant à leur sphère amicale qui ont pris part à leur apprentissage. André a pu, par exemple, utiliser pour la première fois un régulateur de vitesse en conduisant la voiture d'un ami. Ce dernier était « fier » de montrer à André le système innovant présent sur son véhicule. Il lui a également enseigné les rudiments de l'utilisation (objectif du dispositif, emplacement et fonction des commandes). Cet épisode a été répertorié sur la ligne d'usage complétée par André (Figure 3).

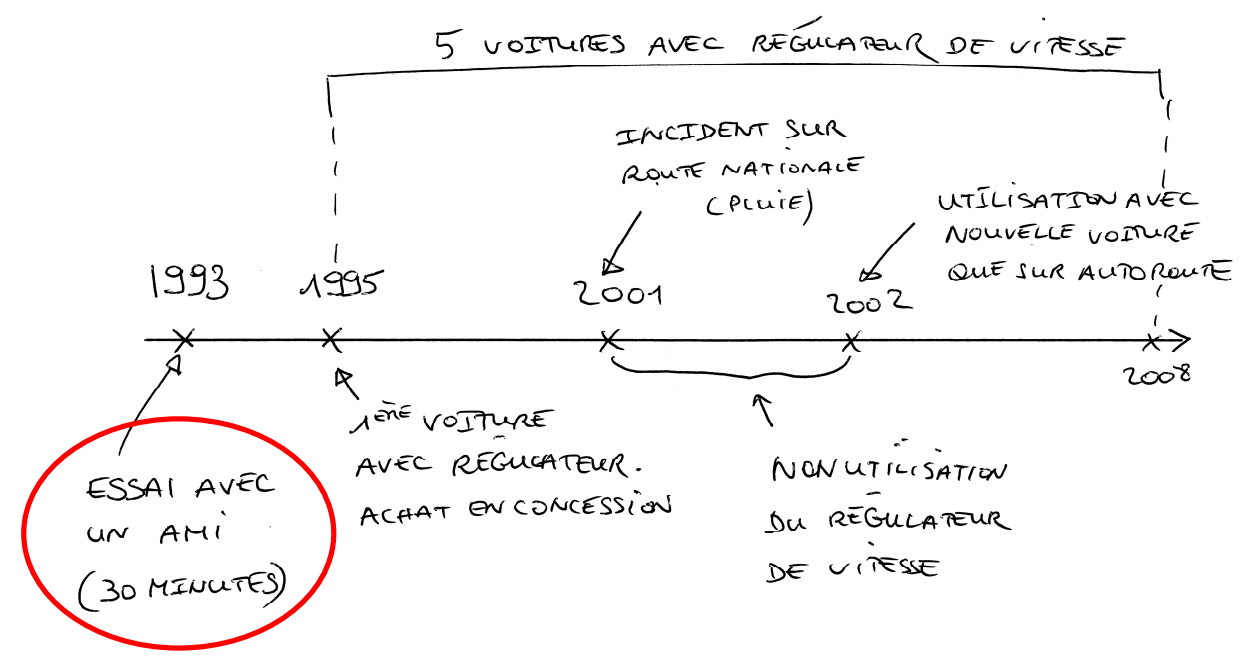

Figure 3. Ligne d'usage du régulateur de vitesse complétée par André 
A travers ces exemples, on peut constater que l'apprentissage de l'utilisation du régulateur de vitesse ne se fait pas seul. D'autres individus de l'entourage du conducteur sont impliqués dans la découverte de son fonctionnement. Il est intéressant de voir à quel point l'enseignement des ces «formateurs informels » est pris en considération par les conducteurs. Ces derniers placent une confiance importante dans leurs proches qui sont assimilés à des experts de l'usage du CCC. Les résultats tendent également à indiquer que les conducteurs reproduisent le rapport qu'entretenait leur formateur principal avec le régulateur de vitesse. Toute une série de questions se pose alors sur le contenu réel de cet enseignement et sur les modalités d'appropriation de ces savoirs de la part du conducteur, compte tenu du fait que ces formateurs, et c'est une hypothèse, ne relayent pas forcément les « bonnes pratiques » d'usage du système. Ce point mérite une analyse plus systématique des situations d'apprentissage en contexte réel.

\subsubsection{L'absence des professionnels des concessions automobiles dans}

\section{l'appropriation du dispositif}

Les entretiens menés avec les conducteurs, pour la plupart acheteurs de voitures neuves (sept sur dix), indiquent le peu d'implication des commerciaux des concessions automobiles dans l'appropriation du régulateur de vitesse. Des extraits d'entretiens menés avec Christophe puis André sont présentés ci-après :

«Le commercial ne m’a pas montré comment utiliser le régulateur! Il a attiré mon attention dessus, normal ça a un aspect commercial. Il m'a dit que j'aurai le régulateur de vitesse sur l'automobile, ça faisait partie de son argumentaire, et ça c'est arrêté là. C'est la seule fois que l'on m'en a parlé à la concession. (...) En fait je n'ai jamais eu d'explication sur le régulateur de la part de la concession. »(Christophe, entretien d'explicitation) 
«Je comprends que les gens qui n’ont jamais eu de régulateur de vitesse trouvent ça difficile à utiliser. La formation est inexistante. J'ai pris ma nouvelle voiture dans une concession et le gars a listé tout un tas de trucs, il n'y avait pas que le régulateur.

On vous liste un tas de trucs...

Oui en 20 ou 30 minutes, le gars il vous dit tout sur la voiture. Après pour le régulateur il faut reprendre la notice mais avec un système comme ça, on ne peut pas le manipuler à l'arrêt. Ce n'est qu'en roulant qu'on voit comment ça fonctionne et comment ça se déconnecte ». (André, entretien d'explicitation)

Les données attestent de l'absence des professionnels des concessions automobiles dans la découverte et l'appropriation du CCC. Les vendeurs recensent les équipements embarqués dans le véhicule sans présenter leurs modes d'usage. Comme le régulateur de vitesse est considéré comme un critère d'achat secondaire face à l'esthétique ou la consommation du véhicule, aucune précision n'est demandée lors de la situation d'achat. A notre avis, des investigations approfondies et prolongées des interactions entre clients et commerciaux pourraient permettre de mieux comprendre cette situation d'achat et repositionner les concessions automobiles dans la formation des conducteurs sur ce point.

\section{DISCUSSION}

Les résultats de cette étude indiquent clairement que d'autres individus que le conducteur (passagers et autres usagers de la route) sont impliqués dans l'usage du régulateur de vitesse conventionnel, parfois même de façon radicale. Ces résultats sont cohérents avec ceux obtenus dans AIDE (Lheureux et coll., 2006 ; Saad et coll., 2006) et les récents travaux portant sur l'ACC (Bjørkly, Jenssen, Moen et Vaa, 2003 ; Rudin-Brown et Parker, 2004 ; Ojeda et Nathan, 2006) mettant en lumière le poids d'une dimension collective dans l'usage 
de ces systèmes. Nos résultats indiquent également que le conducteur apprend à utiliser le régulateur de vitesse conventionnel au contact de plusieurs personnes. Là encore ces résultats se retrouvent dans le prolongement de ceux de Pagot (2010) portant sur le CCC ainsi que ceux de Portouli et Papakostopoulos (2006) et Jenness et coll. (2008) pour l'ACC.

Le niveau d'analyse de la démarche ergonomique proposée ici a ainsi permis (a) d'apporter des éléments de description de l'usage et de l'appropriation effective du régulateur de vitesse, (b) de préciser l'articulation entre l'activité des passagers et le conducteur au cours de la conduite avec ce système, (c) de commencer à identifier des situations à risque provoquées par la cohabitation entre automobilistes équipés/non équipés, (d) de générer un certain nombre d'avancées en termes de connaissances scientifiques et d'application sur les processus psychologiques mis en jeu dans la conduite automobile, (e) de fournir une base pertinente pour l'étude rigoureuse de la cognition humaine dans des activités autres que la conduite automobile, en raison du potentiel de généralisation de cette démarche.

Même si du point de vue de l'accidentologie, une mauvaise utilisation d'un SAC présent dans le véhicule ne semble pas une cause significative d'accidents (d'après les études détaillées d'accidents (EDA) de l'INRETS et la base de données BAAC concernant l'ensemble des accidents corporels français relevé pas les forces de l'ordre), un certain nombre de difficultés sont bien présentes pour les conducteurs : des problèmes liés au confort d'utilisation et des difficultés d'appropriation (même de dispositifs de première génération, comme le régulateur de vitesse conventionnel). Un paradoxe lorsque l'on sait que les efforts des constructeurs et des équipementiers vont dans le sens de produits de plus en plus sophistiqués et diversifiés. 


\section{Remerciements}

Les auteurs tiennent à remercier Luciano Ojeda pour ses commentaires constructifs sur les versions successives de ce manuscrit. 


\section{Références}

AIDE.- Adaptative Integrated Driver-Vehicle Interface, Projet Européen (6ème PCRD), 2004-2008.

Aldridge (Brian), Himmler (Meredith), Aultman-Hall (Lisa), Stamatiadis (Nick).- Impact of passengers on driver safety, Transportation Research Board, 1693, 1999, p. 25-30.

Bachmann (Thomas), Naab (Karl), Reichart (Günter), Scrault (Michael).- Enhancing traffic safety with BMW's driver assistance approach connected drive ATA, Ingeneria Automotoristica, 53, 2000, p. 470-483.

Baxter (James S.), Manstead (Anthony S. R.), Stradling (Stephen G.), Campbell (Karen A.), Reason (James T.), Parker (Diane).- Social facilitation and driver behaviour, British Journal of Psychology, 81, 1990, p. 351-360.

Bjørkly (Cato A), Jenssen (Gunnar D.), Moen (Terje), Vaa (Torgeir).- Adaptive Cruise Control (ACC) and driver performance: effects on objective and subjective measures, 10th World Congress on Intelligent Transport Systems, Madrid, 16-20 nov 2003.

Cacciabue (Pietro Carlo), Saad (Farida).- Behavioural adaptations to driver support systems: a modelling and road safety perspective, Cognition, Technology \& Work, 10, 2008, p. 31-39.

Callahan (Joseph M.).- The cruise man, Automotive Industries, 172, 1992, p. 42-44.

Chen (Li-Hui), Baker (Susan P.), Braver (Elisa R.), Li (Guohua).- Carrying passengers as risk factor for crashes fatal to 16 and 17 year old drivers, Journal of the American Medical Association, 283, 2000, p. 1578-1582.

Connolly (Terry), Aberg (Lars).- Some contagion models of speeding, Accident Analysis and Prevention, 25(1), 1993, p. 57-66.

Delhomme (Patricia).- La présence de passager(s) favorise-t-elle le port de la ceinture de sécurité en ville ?, Bulletin de Psychologie, 418, 1994, p. 38-49. 
Doherty (Sean T.), Andrey (Jean C.), McGregor (Carolyn).- The situational risks of young drivers: the influence of passengers, time of day and day of week on accident rates, Accident Analysis and Prevention, 30, 1998, p. 45-52.

Dominicé (Pierre).- L'histoire de vie comme processus de formation, Paris, L'Harmattan, 2002.

EuroFOT.- European Field Operational Test on Active Safety Systems in vehicles, Projet Européen, 7ème PCRD, 2008-2009.

Fancher (Paul), Ervin (Robert), Sayer (Jim), Hagan (Michael),. Bogard (Scott), Bareket (Zevi), Mefford (Mary), Haugen (Jim).- Intelligent cruise control field operational test, UMTRI, Final Report, 1998.

FESTA.- Field Operational Test Support Action, Projet Européen, 7ème PCRD, 2006-2008.

Glasser (Barney G.), Strauss (Anselm L.).- The discovery of grounded theory, Chicago, Adeline, 1967.

Goodman (Michael J.), Tijerina (Louis), Bents (Frances D.), Wierwille (Walter W).- Using cellular telephones in vehicles: safe or unsafe?, Transportation Human Factors, 1, 1999, p. 342.

Holland (Carol A.), Conner (Mark T.).- Exceeding the speed limit, an evaluation of the effectiveness of a police intervention, Accident Analysis and Prevention, 28(5), 1996, p. 587597.

Hutchins (Edwin).-Cognition in the Wild, Cambridge, The MIT Press, 1995.

Jenness (James W) Lerner (Neil D.), Mazor (Steve), Osberg (Scott), Tefft (Brian, C).- Use of advanced in-vehicle technology by young and older early adopters, DOT HS 811 004, National Highway Traffic Safety Administration (NHTSA), 2008. 
Koziol (Joseph S.), Inman (Vaughn W.), Carter (Mark), Robinson (Mark), Baker (Mark).Evaluation of intelligent cruise control system. Volume 1 - Study results, DOT-VNTSCNHSTA- 98-3, Washington, DC, Department of Transport, 1999.

Lee (John D.), Caven (Brent), Haake (Steven), Brown (Timothy L.).- Speech-based interaction with in-vehicle computers: the effect of speech-based e-mail on drivers' attention to the road, Human Factors, 2001, p. 631-640.

Lheureux (Florent), Saad (Farida)., Pianelli (Carinne), Abric, (Jean-Claude), Roland (Jacques).- Behavioural changes due to long term use of speed limiter and cruise control, dans AIDE project (Adaptive Integrated Driver-vehicle Interface), D1.2.4., Long-term phase test and results, European Commission, Brussels, 2006, p. 39-64.

Mevel (Yannick) (2000). La ligne de vie professionnelle. Les Cahiers Pédagogiques, 380, 4647.

Ojeda (Luciano), Nathan (Florence).- Studying learning phases of an ACC through verbal reports, dans Brouwer (R.F.T.) et Hoedemaeker (D.M.) (Eds.), Driver support and information systems: experiments on learning, appropriation and effects of adaptiveness, AIDE project (Adaptive Integrated Driver-vehicle Interface), D1.2.3. European Commission, Brussels, 2006, p. 47-73.

Pagot (Chloé).- Genèses instrumentales, pouvoir d'agir et capacité d'agir des automobilistes conduisant avec un système d'aide à la régulation longitudinal. Le régulateur de vitesse. Thèse de doctorat en ergonomie, Université Paris 8.

Peretti-Watel (Patrick).- La conduite automobile : un objet de recherche sociologique ? European Journal of Sociology, 42, 2001, p. 391-428.

Portouli (Villy), Papakostopoulos (Vassilis).- On-road study on short-term effects of ADAS on driving behaviour, dans Brouwer (R.F.T.) et Hoedemaeker (D.M.) (Eds.), Driver support and information systems: experiments on learning, appropriation and effects of adaptiveness, 
AIDE project (Adaptive Integrated Driver-vehicle Interface), D1.2.3. European Commission, Brussels, 2006, p. 74-96.

Regan (Michael A.), Mitsopoulos (Eve).- Understanding passenger influences on driver behaviour: implications for road safety and recommendations for countermeasure development, Monash (Australia), Monash University Accident Research Centre, 2003.

Renault.-Guide de mise en main du régulateur/limiteur de vitesse, 2004.

Rolls (Gordon), Ingham (Roger).- Safe and unsafe, a comparative study of younger male drivers, Basingstoke, United Kingdom, AA Foundation for Road Safety Research, 1992.

Rudin-Brown (Christina M.), Parker (Heather A).- Behavioural adaptation to adaptive cruise control (ACC): implications for preventive strategies, Transportation Research, 7, 2004, p. $59-76$.

Saad (Farida), Malaterre (Gilles).- Régulation de la vitesse, aide au contröle de la vitesse, Rapport ONSER, 1982.

Saad (Farida), Villame (Thérèse).- Assessing new driving support system: contribution of an analysis of drivers' activity in real situations, Third annual World Congress on Intelligent Transport Systems, Orlando, USA, 1996.

Saad (Farida), Dionisio (Corinne), Arslanian (Elsa), Colaciuri (Véronique), Legay (Frédéric), Vézier (Bénédicte), Timianguel (Josué).- Learning phase and short term behavioural effect of speed limiter and cruise control systems, dans Brouwer (R.F.T.) et Hoedemaeker (D.M.) (Eds.), Driver support and information systems: experiments on learning, appropriation and effects of adaptiveness, AIDE project (Adaptive Integrated Driver-vehicle Interface), D1.2.3. European Commission, Brussels, 2006, p. 19-46.

Saad (Farida), Hjälmdahl (Magnus), Cañas (José), Alonso (Maria), Garayo (Pedro), Macchi (Luigi), Nathan (Florence), Ojeda (Luciano), Papakostopoulos (Vassilis), Panou (Maria), Bekiaris (Evangelos).- Behavioural effects of driver assistance system, AIDE Project 
(Adaptive Integrated Driver-vehicle Interface), D1.2.1. European Commission, Brussels, 2004.

Shaout (Adnan K.), Jarrah (Mohammad A.).- Cruise control technology review, Computers and Electrical Engineering, 23, 1997, p. 259-271.

Smith (Frank J.), Ashby (Matthew C.), Fairclough (Stephen H.).- Cruise control use: driver attitudes and behaviour, Triennial Congress of the International Ergonomics Association, Toronto, Human Factors Association of Canada, 1994, p. 252-254.

Stanton (Neville A.), Young (Mark S.).- Driver behaviour with adaptive cruise control, Ergonomics, 48(10), 2005, p. 1294-1313.

Stanton (Neville A.), Young (Mark S.), McCaulder (Brian).- Drive-by-wire: the case of driver workload and reclaiming control with adaptive cruise control, Safety Science, 27, 1997, p. $149-159$.

Suchman (Lucy).- Plans and situated action, Cambridge, Cambridge University Press, 1987.

Theureau (Jacques), Jeffroy (François).- Ergonomie des situations informatisées, Toulouse, Octarès, 1994.

Theureau (Jacques).- Le cours d'action, Toulouse, Octarès (2 $2^{\text {ème }}$ éd.), 2004.

Travis (Claude J.).- Cruise control improves fuel mileage, Diesel Equipment Superintendent, 60(4), 1982, p. 40.

Vermersch (Pierre).- Questionner l'action : l'entretien d'explicitation, Psychologie Française, 35(3), 1990, p. 227-235.

Villame (Thérèse), Theureau (Jacques).- Contribution of a 'comprehensive analysis' of human cognitive activity to the advanced driving assistance devices design, dans Onken (R.) (Ed.), Proceedings of CSAPC, $8^{\text {th }}$ Conference on Cognitive Science Approaches to Process Control, Bonn, 2001,p. 23-26. 
Wang (Jing-Shiarn), Knipling (Ronald R.), Goodman (Michael J.).- The role of driver inattention in crashes: new statistics from the 1995 Crashworthiness Data System, $40^{\text {th }}$ annual proceedings of the association for the Advancement of Automotive Medicine, 1996, p. 377392.

Warren (Davey L.).- Speed zoning and control. Synthesis of safety research related to traffic control and roadway elements, Report No. FHWA-TS-82-233, Washington, DC, Department of Transport, 1982.

Young (Kristie L.), Regan (Michael A).- Use of manual speed alerting and cruise control devices by car drivers, Safety Science, 45(4), 2007, p. 473-485.

Youngbin (Yim).- A focus group study of automated highway systems and related technologies, Berkley, California, California PATH Working Paper, Institute of Transport Studies, 1997.

Zaidel (David M.), Paarlberg (William T.), Shinar (David).- Driver performance and individual differences in attention and information processing. Volume 1: Driver inattention, DOT-HS-803 793, Institute for Research in Public Safety, 1978. 